\title{
OGTT and random plasma glucose in the prediction of type 1 diabetes and time to diagnosis
}

\author{
Olli Helminen ${ }^{1}$ - Susanna Aspholm ${ }^{2}$ - Tytti Pokka ${ }^{1}$. Jorma Ilonen ${ }^{3,4}$ • Olli Simell ${ }^{5}$. \\ Riitta Veijola $^{1} \cdot$ Mikael Knip ${ }^{2,6,7,8}$
}

Received: 22 December 2014 / Accepted: 20 April 2015 /Published online: 19 May 2015

(C) Springer-Verlag Berlin Heidelberg 2015

\begin{abstract}
Aims/hypothesis We assessed the utility of the OGTT and random plasma glucose concentrations in predicting the time to diagnosis of type 1 diabetes.

Methods A population-derived cohort of 14,876 newborns with HLA-conferred risk of type 1 diabetes were invited to regular follow-up for islet autoantibodies. When two or more autoantibodies were detected, an OGTT was performed once a year and random plasma glucose analysed twice a year. During follow-up, 567 children developed multiple autoantibodies, 255 (45\%) of whom were diagnosed with type 1 diabetes, while 312 remained non-diabetic by December 2011.
\end{abstract}

Olli Helminen and Susanna Aspholm contributed equally as first authors. Riitta Veijola and Mikael Knip contributed equally as senior authors.

Olli Helminen

olli.helminen@oulu.fi

1 Department of Pediatrics, PEDEGO Research Group, Medical Research Center, Oulu University Hospital and University of Oulu, PO Box 5000, FIN-90014 Oulu, Finland

2 Tampere Centre for Child Health Research, Tampere University Hospital, Tampere, Finland

3 Immunogenetics Laboratory, University of Turku, Turku, Finland

4 Department of Clinical Microbiology, University of Eastern Finland, Kuopio, Finland

5 Department of Pediatrics, University of Turku and Turku University Hospital, Turku, Finland

6 Children's Hospital, University of Helsinki and Helsinki University Hospital, Helsinki, Finland

7 Research Programs Unit, Diabetes and Obesity, University of Helsinki, Helsinki, Finland

8 Folkhälsan Research Center, Helsinki, Finland
Results Impaired fasting glucose (IFG) and impaired glucose tolerance (IGT) were risk factors for type 1 diabetes (HR 3.2 [95\% CI 1.5, 7.0] and 8.3 [95\% CI 6.0, 11.5], respectively). When a random plasma glucose value $\geq 7.8 \mathrm{mmol} / 1$ was observed, the HR for diabetes was $6.0(95 \%$ CI $4.3,8.6)$. The median time to diagnosis after the detection of IFG was 5.2 years (interquartile range [IQR] 3.4, 6.3); after IGT, 0.7 years (IQR $0.3,1.9$ ); and, after a random plasma glucose $\geq 7.8 \mathrm{mmol} / \mathrm{l}, 1.0$ years (IQR $0.3,1.5$ ). In a retrospective analysis, both OGTT-derived $2 \mathrm{~h}$ plasma glucose and random plasma glucose started to increase 1.5 years before diagnosis $(p<0.001$ and $p=0.004$, respectively).

Conclusions/interpretation Dysglycaemia detected in an OGTT or based on random plasma glucose is a useful marker in the prediction of time to onset of type 1 diabetes in high-risk children. Random plasma glucose is a simple and low-cost measurement with comparable predictive characteristics to that of OGTT-derived $2 \mathrm{~h}$ glucose.

Keywords Children · Dysglycaemia $\cdot$ Islet autoimmunity · OGTT $\cdot$ Plasma glucose $\cdot$ Type 1 diabetes

\begin{tabular}{ll}
\multicolumn{2}{l}{ Abbreviations } \\
DIPP & Type 1 Diabetes Prediction and Prevention Project \\
DPT & Diabetes Prevention Trial \\
GADA & GAD65 antibodies \\
IAA & Insulin autoantibody \\
IA-2A & Insulinoma-associated antigen-2 antibody \\
ICA & Islet cell antibody \\
IFG & Impaired fasting glucose \\
IGT & Impaired glucose tolerance \\
IQR & Interquartile range
\end{tabular}




\section{Introduction}

Type 1 diabetes is one of the most common chronic diseases in childhood. The annual increase in incidence has been reported to be approximately $3.0 \%$ worldwide [1]. The highest incidence has been observed in Finland, where the rate more than doubled between the years 1980 and 2005, especially among children under the age of 5 [2]. The presentation of type 1 diabetes is typically acute, although a decline in beta cell function begins several years before the appearance of symptoms. Currently, type 1 diabetes is predicted by analysing disease-associated islet autoantibodies in children with increased genetic susceptibility. Children with multiple islet autoantibodies and risk-conferring HLA class II genotypes have a cumulative disease risk of $50-60 \%$ over the next 5 years and a more than $80 \%$ risk when followed for 15 years after seroconversion to autoantibody positivity [3, 4]. Although abnormalities in glucose metabolism and insulin secretion are present years before diagnosis, prediction of the timing of clinical disease has remained challenging [5-10].

The diagnosis of type 1 diabetes is often made early among children who participate in prospective follow-up studies. This has resulted in a marked reduction in the incidence of diabetic ketoacidosis at diagnosis compared with children diagnosed in the background population [11, 12], and also in a reduced risk of complications during the first year after diagnosis [13]. Accordingly, early detection of clinical disease is advantageous.

The OGTT is the gold standard for diagnosing diabetes [14]. There is evidence that impaired fasting glucose (IFG) and impaired glucose tolerance (IGT) often precede clinical diagnosis $[15,16]$. In this study we set out to assess how often dysglycaemia detected by OGTT occurred in a populationderived cohort of children with HLA-conferred disease susceptibility and multiple (two or more) islet autoantibodies, and to evaluate the potential of IFG and IGT in predicting time to onset of clinical type 1 diabetes. In addition, we have characterised for the first time the behaviour and predictive characteristics of random plasma glucose concentrations in the same cohort of children with multiple islet autoantibodies.

\section{Methods}

Study design The Type 1 Diabetes Prediction and Prevention Project (DIPP) is a Finnish birth cohort study which started recruitment of newborn babies in November 1994 and continues recruitment at three university hospitals (Turku, Oulu and Tampere). Cord blood samples from 168,055 newborn infants were screened for HLA-conferred susceptibility to type 1 diabetes until December 2011. Of the families with a susceptible infant, 14,876 decided to join the prospective follow-up at 3-12 month intervals until a diagnosis of type 1 diabetes or the age of 15 years. The study has been approved by the ethics committees in the participating universities and hospital districts. All families participating in the study have provided written informed consent.

Islet autoantibodies were analysed at each visit and monitoring of random plasma glucose and an OGTT were initiated in children who tested positive for at least two of the autoantibodies in the same sample. Random plasma glucose concentrations were analysed at every visit, except when an OGTT was carried out. An OGTT was performed once a year. The diagnosis of type 1 diabetes was based on typical symptoms and high random plasma glucose levels; or in an asymptomatic patient, diagnostic plasma glucose values in two separate OGTTs as recommended by the WHO [14]. The inclusion criteria for the current analysis comprised all children with multiple (two or more) islet autoantibodies who had at least one random plasma glucose analysed and/or at least one OGTT performed by 31 December 2011.

A total of 567 children were included in the study. The inclusion criteria were fulfilled by 505 children in the random plasma glucose series and by 403 children in the OGTT series. In the random plasma glucose series, 204 children were eventually diagnosed with type 1 diabetes (progressors) before the end of follow-up, whereas 301 remained disease-free (nonprogressors). The OGTT series comprised 209 progressors and 194 non-progressors.

Immunological screening Participants who seroconverted to positivity for any of the diabetes-associated islet autoantibodies analysed, i.e. islet cell antibodies (ICA), insulin autoantibodies (IAA), GAD65 antibodies (GADA) or insulinomaassociated antigen-2 antibodies (IA-2A), were rescheduled for follow-up visits at 3 month intervals. Autoantibody seroconversion was defined as the time when at least one autoantibody was detected for the first time, and seroconversion to positivity for multiple autoantibodies as the time when at least two autoantibodies were detected in the same sample for the first time. Positive results were confirmed in a subsequent sample. Primary islet autoantibody was defined as the first detected autoantibody, or the first combination if multiple islet autoantibodies occurred in the same sample. Autoantibodies were analysed as described previously [3]. ICA titres were measured using a standard indirect immunofluorescence assay with a detection limit of 2.5 Juvenile Diabetes Foundation units. GADA levels were analysed using a radiobinding assay with a cut-off limit for positivity of 5.36 relative units. IAA levels were measured using a modified radiobinding assay with a cut-off limit of 3.48 relative units. IA-2A levels were also quantified with a radiobinding assay, the cut-off limit being 0.43 relative units. In the Diabetes Autoantibody Standardization Program workshop in 2005 the following sensitivities and specificities were reported: IAA 58\% and 96\%, 
GADA $82 \%$ and $96 \%$, and IA-2A $72 \%$ and $100 \%$, respectively.

Genetic screening HLA-conferred susceptibility to type 1 diabetes was analysed centrally using cord blood as described earlier [17]. According to various HLA-DRB1-DQA1DQB1 haplotype combinations, genotypes conferring high, moderate and low risk of type 1 diabetes were determined. High risk was defined as heterozygosity for the two riskassociated haplotypes $D R B 1 * 04: 01 / 2 / 4 / 5 / 8-D Q A 1 * 03$ $D Q B 1^{*} 03: 02 / 4 / D R B 1 * 03-D Q A 1 * 05-D Q B 1 * 02$. Moderate risk was defined as homozygosity for any of these two risk haplotypes or $D R B 1^{*} 04: 01 / 2 / 4 / 5 / 8-D Q A 1^{*} 03-D Q B 1^{*} 03: 02 /$ 4 combined with a neutral haplotype or the $D R B 1^{*} 03$ $D Q A 1 * 05-D Q B 1 * 02 / D R B 1 * 09-D Q A 1 * 03-D Q B 1 * 03$ genotype. Low risk was conferred by other genotypes as previously defined [18].

OGTTs and glucose assays Oral glucose $(1.75 \mathrm{~g} / \mathrm{kg}$ body weight, up to a maximum of $75 \mathrm{~g}$ ) was administered after overnight fasting. Samples were taken at 0 and $120 \mathrm{~min}$ and tested for glucose in the local laboratory. In Oulu University Hospital capillary samples were used, whereas in Tampere and Turku tests were based on venous samples. In Oulu and Tampere whole blood was used until May 2000 and 2003, respectively, and thereafter plasma was analysed. Only $0.7 \%$ of the samples were obtained from whole blood. In Oulu University Hospital a glucose dehydrogenase-based method was used until May 2000, and thereafter a glucose oxidase method was used. In Tampere and Turku the hexokinase method was applied during the whole study. Statistical analyses considering methodological differences are presented in detail in the statistical analyses section.

Random plasma glucose assays Random plasma glucose measurements were carried out in the three participating university hospitals of Oulu, Tampere and Turku. Samples were obtained from venous plasma in all study sites, except for samples taken in Oulu University Hospital before May 2000 and Tampere University Hospital before May 2003, where whole blood samples were used (only $0.5 \%$ of samples). Oulu used the glucose dehydrogenase method until May 2000 and continued with the enzymatic glucose hexokinase method. Tampere used a hexokinase-based method until September 2006 and subsequently a glucose dehydrogenase method was applied. In Turku the glucose dehydrogenase method was used during the whole follow-up. Methodological differences have been taken into account in the analyses (see the statistical analyses section).

Definition of dysglycaemia Glucose tolerance status in the OGTT was defined according to the WHO recommendation, taking into account the different cut-offs for the methods used, and categorised as normal, IFG, IGT and diabetic [19]. In order to compare the results of random plasma glucose and standardised OGTT, the random plasma glucose series was categorised only as normal or $\geq 7.8 \mathrm{mmol} / \mathrm{l}$, the cut-off being the same as that used for IGT during a standardised OGTT. Diabetes was diagnosed according to the WHO criteria as mentioned earlier [14].

Statistical analyses In the OGTT series, three different variables were analysed and the children were analysed in three different groups: IFG or not (decision rule 1), IGT or not (decision rule 2), and both IFG and IGT or not (decision rule 3). The applied variables were based on the WHO recommendations.

In the random plasma glucose series, a single variable was analysed: whether children experienced a single random plasma glucose value $\geq 7.8 \mathrm{mmol} / 1$ or not (decision rule 4 ). No cutoff values for randomly taken plasma glucose have been proposed for the prediction of type 1 diabetes, and we chose the value of $7.8 \mathrm{mmol} / \mathrm{l}$ according to the WHO criteria for dysglycaemia during OGTT.

The difference in the proportions of dysglycaemia in various primary islet autoantibody groups was tested with the standardised normal deviate test. ICA were always associated with biochemical islet autoantibodies during follow-up and were excluded from this analysis. Eventually seven different primary islet autoantibody groups were defined.

Cox regression with time-dependent covariates was used to evaluate the association between glucose variables and the risk of diabetes. The entry time to the analysis was the date when at least two islet autoantibodies were positive. All participants were considered to be unexposed until the timedependent covariate fulfilled the decision rule, and thereafter the status of the participant was exposed. The total follow-up time was partitioned into intervals according to cut-off points of the decision rules. Univariate Cox regression analysis with survival curve estimates was performed to assess the risk and timing of diabetes after fulfilling a decision rule. Adjusted HRs with inclusion of the following confounding factors were also calculated: sex, age at multiple (two or more) autoantibodies, time from seroconversion to multiple (two or more) autoantibodies, type 1 diabetes in a first-degree relative and HLA risk. Samples taken on the day of diagnosis were excluded from the Cox regression analyses. Sensitivity and specificity of the four decision rules were also calculated.

The linear mixed model with random intercept and diagonal covariance structure for repeated measurements was used to analyse OGTT and random plasma glucose levels over time between the progressors and non-progressors. The random intercepts and repeated measurements were nested within participants and participants within hospital. The group-by-time interaction was included in the model to test differences between group means at each time point. Sex, age at sampling, 
age at multiple (two or more) autoantibodies, time from seroconversion to multiple (two or more) autoantibodies, an affected first-degree relative and HLA risk were included in the linear mixed model as fixed variables.

All analyses were performed using IBM SPSS Statistics for Windows (version 22.0; SPSS, Chicago, IL, USA) and Stata IC version 13.1 for Windows (StataCorp LP, College Station, TX, USA). Figures were drawn using OriginPro 9.1.0 (OriginLab, Northampton, MA, USA) and Stata IC version 13.1.

\section{Results}

Between November 1994 and December 2011, 14,876 infants with increased genetic risk were enrolled for regular follow-up in the DIPP. During follow-up 567 children developed multiple (two or more) islet autoantibodies, of whom 255 (45\%) progressed to clinical disease, whereas $312(55 \%)$ remained non-diabetic. In the group of progressors the participants developed multiple (two or more) autoantibodies at a mean age of 2.9 years (SD 2.2), whereas the mean age in the nonprogressor group was 5.4 years (SD 3.5). We analysed the data in two series, the OGTT series with 403 participants and the random plasma glucose series with 505 participants. Three hundred and forty-one children participated in both series. The basic characteristics of the study participants are presented in Table 1.

Altogether 1,403 OGTTs were carried out during followup, of which 697 were in the progressors and 706 in the nonprogressors. A mean of 3.6 tests were performed per child (range 1-15). In the random plasma glucose series, 3,435 samples were analysed for plasma glucose: 1,201 in the progressors and 2,234 in the non-progressors. A mean of 6.8 samples per child were analysed (range 1-29).

OGTTs We tested the impact of dysglycaemia with Cox regression analysis and identified significant predictors of type 1 diabetes (Table 2). IFG, IGT and their combination all showed high and significant HRs. For the whole study population of children with multiple islet autoantibodies the median diabetes-free survival time was 6.9 years (Fig. 1a). The median time to type 1 diabetes after observed IFG was 5.2 years (IQR 3.4, 6.3; Fig. 1b); and after IGT, 0.7 years (IQR 0.3, 1.9; Fig. 1c).

When retrospectively comparing the glucose concentrations in OGTT between progressors and non-progressors we observed that the $2 \mathrm{~h}$ values started to be consistently higher in the progressors 1.5 years before diagnosis ( $p<0.001$; Fig. $2 b$ ). More specifically, during the period 1.5-2 years before diagnosis the adjusted mean of $2 \mathrm{~h}$ glucose was $5.9 \mathrm{mmol} / \mathrm{l}(95 \%$ CI 5.6, 6.3) in the progressors compared with $5.5 \mathrm{mmol} / \mathrm{l}$ $(95 \%$ CI $5.0,6.0)$ in the non-progressors $(p=0.113)$. During

Table 1 Baseline characteristics of the study population

\begin{tabular}{|c|c|c|c|c|}
\hline \multirow[t]{2}{*}{ Characteristic } & \multicolumn{2}{|l|}{ OGTT } & \multicolumn{2}{|c|}{ Random plasma glucose } \\
\hline & $\begin{array}{l}\text { Progressors }^{\mathrm{a}} \\
N=209\end{array}$ & $\begin{array}{l}\text { Non-progressors }{ }^{\mathrm{b}} \\
N=194\end{array}$ & $\begin{array}{l}\text { Progressors }^{\mathrm{a}} \\
N=204\end{array}$ & $\begin{array}{l}\text { Non-progressors }{ }^{\mathrm{b}} \\
N=301\end{array}$ \\
\hline \multicolumn{5}{|l|}{ Sex, $n(\%)$} \\
\hline Boys & $124(59)$ & $119(61)$ & $116(57)$ & $194(65)$ \\
\hline Girls & $85(41)$ & $75(39)$ & $88(43)$ & $107(35)$ \\
\hline \multicolumn{5}{|l|}{ Type 1 diabetes in first-degree relative at time of birth, $n(\%)$} \\
\hline No & $177(85)$ & $179(92)$ & $171(84)$ & $280(93)$ \\
\hline Yes & $32(15)$ & $15(8)$ & $33(16)$ & $21(7)$ \\
\hline \multicolumn{5}{|l|}{ HLA risk ${ }^{\mathrm{c}}, n(\%)$} \\
\hline Low & $22(11)$ & $21(11)$ & $21(10)$ & $42(14)$ \\
\hline Moderate & $119(57)$ & $131(68)$ & $114(56)$ & $198(66)$ \\
\hline High & $68(33)$ & $42(22)$ & $69(34)$ & $60(20)$ \\
\hline Age at seroconversion, years, mean (SD) & $2.5(2.0)$ & $3.9(2.7)$ & $2.4(1.9)$ & $4.1(2.9)$ \\
\hline Age at multiple (two or more) autoantibodies, years, mean (SD) & $3.0(2.1)$ & $5.2(3.4)$ & $2.9(2.1)$ & $5.5(3.5)$ \\
\hline $\begin{array}{l}\text { Age at diagnosis of type } 1 \text { diabetes or age of non-progressors at } \\
\text { last measurement }{ }^{\mathrm{d}} \text {, years, mean (SD) }\end{array}$ & $6.5(3.2)$ & $8.9(3.9)$ & $6.5(3.3)$ & $11.1(4.1)$ \\
\hline Follow-up time, years, mean (SD) & $3.6(2.5)$ & $6.5(3.6)$ & $3.6(2.6)$ & $5.6(3.2)$ \\
\hline
\end{tabular}

${ }^{\text {a }}$ Progressors are children with multiple (two or more) autoantibodies who progressed to overt type 1 diabetes

${ }^{\mathrm{b}}$ Non-progressors tested positive for multiple autoantibodies but did not develop clinical disease during follow-up

${ }^{\mathrm{c}}$ One non-progressor child carried a rare HLA genotype that was not possible to define

${ }^{\mathrm{d}}$ The follow-up ended by the end of December 2011 
Table 2 Cox regression HRs for the contribution of dysglycaemia detected by OGTT or by random plasma glucose measurement to the progression of type 1 diabetes

\begin{tabular}{|c|c|c|c|c|c|c|c|c|}
\hline Dysglycaemia & Progressors $^{\mathrm{a}}$ & Non-progressors ${ }^{\mathrm{b}}$ & HR & $95 \% \mathrm{CI}$ & $p$ value & Adjusted $\mathrm{HR}^{\mathrm{c}}$ & $95 \% \mathrm{CI}$ & $p$ value \\
\hline \multicolumn{9}{|l|}{ OGTT } \\
\hline \multicolumn{9}{|l|}{$\mathrm{IFG}^{\mathrm{d}}$} \\
\hline No, $n(\%)$ & $107(94)$ & $176(98)$ & 1 & & & & & \\
\hline Yes, $n(\%)$ & $7(6)$ & $4(2)$ & 3.2 & $1.5,7.0$ & 0.003 & 3.1 & $1.4,6.8$ & 0.004 \\
\hline \multicolumn{9}{|l|}{$\mathrm{IGT}^{\mathrm{d}}$} \\
\hline No, $n(\%)$ & $107(65)$ & $176(95)$ & 1 & & & & & \\
\hline Yes, $n(\%)$ & $58(35)$ & $10(5)$ & 8.3 & $6.0,11.5$ & $<0.001$ & 8.7 & $6.3,12.2$ & $<0.001$ \\
\hline \multicolumn{9}{|l|}{ IFG and IGT } \\
\hline No, $n(\%)$ & $107(95)$ & $176(99)$ & 1 & & & & & \\
\hline Yes, $n(\%)$ & $5(5)$ & $1(1)$ & 53.5 & $17.1,167$ & $<0.001$ & 46.6 & $14.8,147$ & $<0.001$ \\
\hline \multicolumn{9}{|c|}{ Random plasma glucose } \\
\hline \multicolumn{9}{|c|}{ Random plasma glucose $\geq 7.8 \mathrm{mmol} / 1$} \\
\hline No, $n(\%)$ & $161(79)$ & $283(94)$ & 1 & & & & & \\
\hline Yes, $n(\%)$ & $43(21)$ & $18(6)$ & 6.0 & $4.3,8.6$ & $<0.001$ & 6.1 & $4.3,8.7$ & $<0.001$ \\
\hline
\end{tabular}

${ }^{\text {a }}$ Progressors are children with multiple (two or more) autoantibodies who progressed to overt type 1 diabetes; $N=209$ for OGTT and 204 for random plasma glucose

${ }^{\mathrm{b}}$ Non-progressors had multiple autoantibodies but did not develop clinical disease during follow-up; $N=194$ for OGTT and 301 for random plasma glucose

${ }^{\mathrm{c}}$ HR was adjusted with sex, age at multiple (two or more) autoantibodies, time from seroconversion to multiple (two or more) autoantibodies, type 1 diabetes in a first-degree relative and HLA risk

${ }^{\mathrm{d}}$ The 'Yes' group was defined as no other abnormalities in the OGTT observed earlier; the 'No'group was defined as no abnormalities found in the OGTT

the following year a significant difference was consistently observed between the two groups, and thereafter the adjusted mean $2 \mathrm{~h}$ glucose in the progressors started to increase more steeply. At the time of diagnosis or the last visit, the adjusted mean $2 \mathrm{~h}$ glucose was $17.8 \mathrm{mmol} / \mathrm{l}(95 \% \mathrm{CI} 17.0,18.5)$ in the progressors compared with $5.4 \mathrm{mmol} / 1$ (95\% CI 5.1, 5.8) in the non-progressors ( $p<0.001$; Fig. 2b). Fasting plasma glucose in the OGTT showed no difference between progressors and non-progressors any earlier than in the last OGTT before diagnosis, when the mean fasting glucose was $6.3 \mathrm{mmol} / \mathrm{l}$ (95\% CI 6.0, 6.6) among the progressors compared with $4.8 \mathrm{mmol} / 1(95 \%$ CI $4.6,4.9 ; p<0.001$; Fig. $2 \mathrm{a})$ in the corresponding OGTT among the non-progressors.

Random plasma glucose The mean random plasma glucose during the entire follow-up was $6.1 \mathrm{mmol} / 1$ (95\% CI 5.9, 6.4) in the progressors and $5.2 \mathrm{mmol} / \mathrm{l}(95 \% \mathrm{CI} 5.2,5.2)$ in the non-progressors. The 90 th percentile for the whole study cohort was $7.4 \mathrm{mmol} / 1$ (progressors $7.8 \mathrm{mmol} / 1$, non-progressors $6.2 \mathrm{mmol} / \mathrm{l})$. If the child presented a random plasma glucose $\geq 7.8 \mathrm{mmol} / \mathrm{l}$, the HR for the development of type 1 diabetes was $6.0(95 \%$ CI $4.3,8.6)$ and the median time to diagnosis was 1.0 year (IQR 0.3, 1.5; Fig. 1d).

The retrospective comparison of random plasma glucose concentrations between progressors and non-progressors showed a significant difference starting also from 1.5 years before diagnosis ( $p=0.004$; Fig. $2 \mathrm{c}$ ). From 1.5 to 2.0 years before diagnosis the adjusted mean random plasma glucose was $5.2 \mathrm{mmol} / 1(95 \%$ CI 5.0, 5.4) in the progressors compared with a mean of $5.1 \mathrm{mmol} / 1(95 \% \mathrm{CI} 4.9,5.3)$ in the nonprogressors $(p=0.400)$. Thereafter, randomly measured plasma glucose increased towards the diagnosis, when the adjusted mean reached $18.6 \mathrm{mmol} / \mathrm{l}(95 \%$ CI $17.6,19.6)$ among the progressors compared with $5.1 \mathrm{mmol} / 1$ (95\% CI 4.6, 5.6) among the non-progressors at their last visit $(p<0.001)$.

Sensitivity and specificity Sensitivities and specificities were calculated for the suggested decision rules. IFG provided a sensitivity of $6 \%(95 \%$ CI $3 \%, 12 \%)$ and a specificity of 98\% (95\% CI 94\%, 99\%), whereas IGT showed a sensitivity of $35 \%(95 \%$ CI $28 \%, 43 \%)$ and a specificity of $95 \%(95 \%$ CI $90 \%, 99 \%$ ). Random plasma glucose $\geq 7.8 \mathrm{mmol} / \mathrm{l}$ gave a sensitivity of $21 \%(95 \%$ CI $16 \%, 27 \%)$ and a specificity of $94 \%$ (95\% CI 91\%, 96\%).

Primary islet autoantibody status and the association with dysglycaemic markers The distribution of IFG, IGT and a random plasma glucose $\geq 7.8 \mathrm{mmol} / 1$ in the seven groups of children with different primary autoantibody profiles is presented in Table 3. In the OGTT series, children with GADA as the primary islet autoantibody developed IGT significantly less often compared with children with IAA: 9.0\% (10/111) 
Fig. 1 Cox regression survival curves showing time to diagnosis of type 1 diabetes after detection of two or more islet autoantibodies. The four panels show survival curves for study children with (a) two or more islet autoantibodies, (b) IFG in OGTT (solid line) or not (dashed line), (c) IGT in OGTT (solid line) or not (dashed line), (d) single random plasma glucose value $\geq 7.8 \mathrm{mmol} / 1$ (solid line) or not (dashed line). Median diabetesfree survival time is indicated for each curve. a

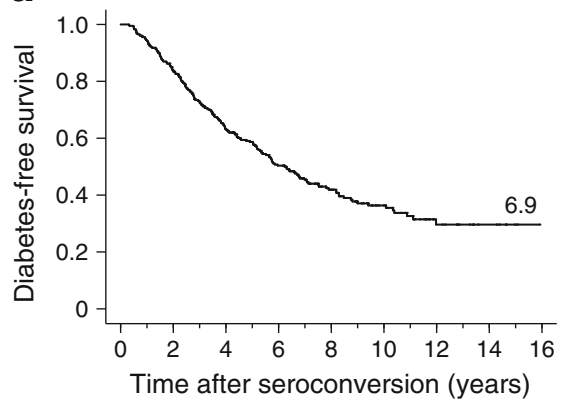

C

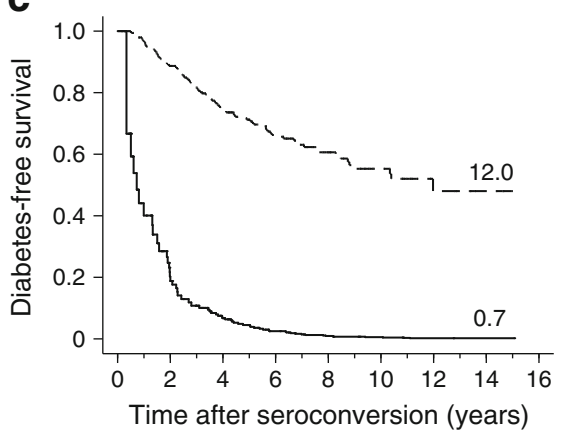

b

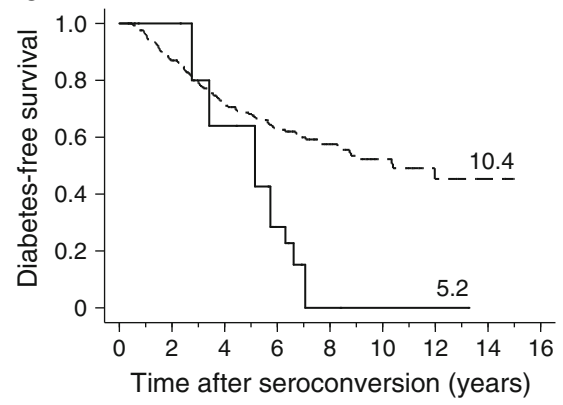

d

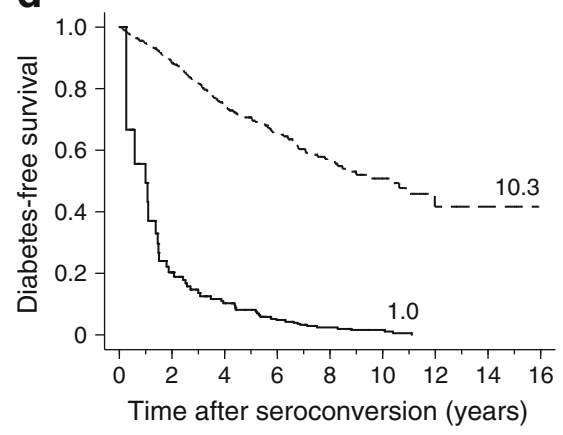

vs $18 \%(21 / 117) ; p=0.038$. A trend for lower occurrence of IGT was observed when comparing children with GADA with those with IA-2A as the initial autoantibody: $9.0 \%$ (10/111) vs
$22 \%(6 / 27) ; p=0.052$. In the random plasma glucose series, children with GADA as the primary autoantibody presented less often with random plasma glucose $\geq 7.8 \mathrm{mmol} / 1$ compared

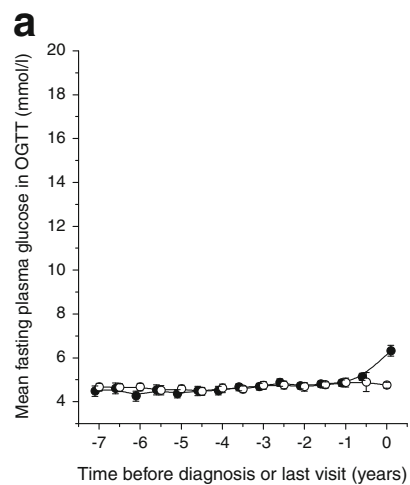

d

\begin{tabular}{lrrrrrrrr} 
Number of OGTTs & & & & & & & \\
\hline Time & -7 & -6.5 & -6 & -5.5 & -5 & -4.5 & -4 & -3.5 \\
\hline Progressors & 7 & 9 & 10 & 17 & 21 & 20 & 28 & 34 \\
Non-progressors & 17 & 21 & 21 & 18 & 26 & 32 & 27 & 44 \\
\hline Time & -3 & -2.5 & -2 & -1.5 & -1 & -0.5 & 0 & \\
\hline Progressors & 43 & 47 & 72 & 60 & 96 & 100 & 35 & \\
Non-progressors & 27 & 50 & 32 & 70 & 43 & 12 & 194 & \\
\hline
\end{tabular}

Fig. 2 Adjusted mean plasma glucose levels in OGTT (fasting and $2 \mathrm{~h}$ plasma glucose) and random plasma glucose series during follow-up in children with multiple $(\geq 2)$ islet autoantibodies. In the linear mixed model analysis, plasma glucose values were adjusted for sex, age at sampling, age at multiple $(\geq 2)$ autoantibodies, time from seroconversion to multiple $(\geq 2)$ autoantibodies, type 1 diabetes in a first-degree relative and HLA risk. The last points are diagnosis of type 1 diabetes (progressors, black circles) or the last follow-up visit by 31 December 2011 (non-progressors, white circles). Whiskers show $95 \%$ CIs of the adjusted mean. (a) No

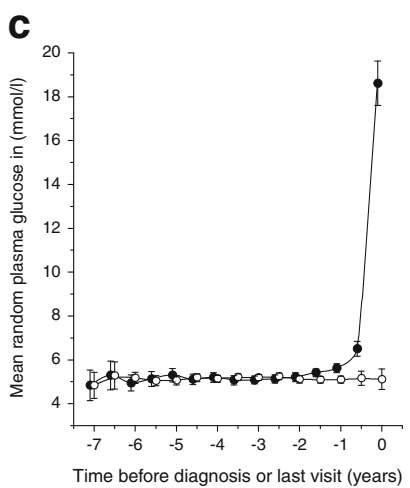

e

\begin{tabular}{lrrrrrrrr} 
Number of random plasma glucose measurements \\
\hline Time & -7 & -6.5 & -6 & -5.5 & -5 & -4.5 & -4 & -3.5 \\
\hline Progressors & 10 & 17 & 19 & 23 & 31 & 43 & 47 & 55 \\
Non-progressors & 14 & 19 & 42 & 54 & 71 & 93 & 109 & 131 \\
\hline Time & -3 & -2.5 & -2 & -1.5 & -1 & -0.5 & 0 & \\
\hline Progressors & 68 & 78 & 92 & 112 & 117 & 113 & 62 & \\
Non-progressors & 146 & 153 & 171 & 178 & 200 & 128 & 301 & \\
\hline
\end{tabular}

difference was observed in the fasting glucose concentrations before the time of diagnosis. (b) The $2 \mathrm{~h}$ plasma glucose values in the OGTT started to be significantly and consistently higher in the progressors 1.5 years before diagnosis. (c) In the random plasma glucose series a significant difference between progressors and non-progressors was also observed 1.5 years before diagnosis. The number of participants at each time point varied during the follow-up but was high in both the OGTT (d) and random plasma glucose series (e), generating reliable results during the whole study period 
Table 3 Distribution of primary islet autoantibody profiles at seroconversion in children in the OGTT and random plasma glucose series. Frequency of various types of dysglycaemia detected during follow-up is presented. Dysglycaemia is defined as IFG or IGT in an OGTT, or by a random plasma glucose value $\geq 7.8 \mathrm{mmol} / 1$. All children developed multiple (two or more) islet autoantibodies during follow-up

Variable

Primary islet autoantibody profile at seroconversion

\begin{tabular}{|c|c|c|c|c|c|c|c|c|}
\hline & \\
\hline & IAA & GADA & IA- $2 \mathrm{~A}$ & $\begin{array}{l}\text { IAA } \\
+ \text { GADA }\end{array}$ & $\begin{array}{l}\text { IAA } \\
+ \text { IA-2A }\end{array}$ & $\begin{array}{l}\text { GADA } \\
+ \text { IA-2A }\end{array}$ & $\begin{array}{l}\text { IAA } \\
+ \text { GADA } \\
+ \text { IA-2A }\end{array}$ & Total $^{\mathrm{a}}$ \\
\hline OGTT series, $n(\%)$ & $117(29)$ & $111(28)$ & $27(7)$ & $66(16)$ & $20(5)$ & $23(6)$ & $39(10)$ & $403(100)$ \\
\hline IFG, $n(\%)$ & $3(2.6)$ & $3(2.7)$ & $1(3.7)$ & $1(1.5)$ & $3(15)$ & $0(0)$ & $0(0)$ & 11 \\
\hline IGT, $n(\%)$ & $21(18)^{b}$ & $10(9.0)^{\mathrm{b}, \mathrm{c}}$ & $6(22)^{\mathrm{c}}$ & $10(15)$ & $5(25)$ & $6(26)$ & $10(26)$ & 68 \\
\hline IFG+IGT, $n(\%)$ & $0(0)$ & $2(1.8)$ & $0(0)$ & $1(1.5)$ & $3(15)$ & $0(0)$ & $0(0)$ & 6 \\
\hline Random plasma glucose series, $n(\%)$ & $158(31)$ & $160(32)$ & $36(7)$ & $73(14)$ & $23(5)$ & $18(4)$ & $37(7)$ & $505(100)$ \\
\hline Random plasma glucose $\geq 7.8 \mathrm{mmol} / 1, n(\%)$ & $18(11)$ & $14(8.8)^{\mathrm{d}}$ & $7(19)^{\mathrm{d}}$ & $10(14)$ & $5(22)$ & $2(11)$ & $5(14)$ & 61 \\
\hline
\end{tabular}

${ }^{\mathrm{a}}$ IAA, GADA and IA-2A in the first positive sample are presented; ICA were always associated with other islet autoantibodies and were not included in this analysis

${ }^{\mathrm{b}}$ IGT was observed less often in the GADA group than in the IAA group $(p=0.038)$

${ }^{\mathrm{c}}$ IGT was observed less often in the GADA group than in the IA-2A group $(p=0.052)$

${ }^{\mathrm{d}}$ Random plasma glucose $\geq 7.8 \mathrm{mmol} / 1$ was observed less often in the GADA group than in the IA-2A group $(p=0.047)$

with children with IA-2A: $8.8 \%(14 / 160)$ vs $19 \%(7 / 36)$; $p=0.047$. There was no significant difference when comparing children with GADA with those with IAA in the development of increased random plasma glucose: $8.8 \%(14 / 160)$ vs $11 \%(18 / 158) ; p=0.365$.

\section{Discussion}

The prediction of type 1 diabetes has so far been based mainly on the presence of islet autoantibodies in individuals at increased genetic risk [3, 20, 21]. In this study we assessed for the first time the development of dysglycaemia in an OGTT to predict the presentation of type 1 diabetes in a populationderived cohort of autoantibody-positive children. Furthermore, the potential impact of randomly measured plasma glucose concentrations was evaluated for the first time in highrisk children.

Our results from the Cox regression analyses indicate that dysglycaemic markers detected by OGTT or random plasma glucose measurement are strong individual predictors of type 1 diabetes. However, the sensitivity of these markers was modest, since IFG was detected in only $6 \%$ and IGT in 35\% of participants before diagnosis. In previous studies with OGTTs performed twice a year, dysglycaemia occurred in $60 \%$ of the study population, suggesting that a test interval of 1 year is too long [15]. Surprisingly, our results show that a randomly taken single plasma glucose appears to predict the presentation of type 1 diabetes almost as well as a standardised OGTT, but again with relatively poor sensitivity (21\%). Age at seroconversion to positivity for multiple (two or more) islet autoantibodies, time from initial seroconversion to multiple autoantibodies, presence of an affected first-degree relative and the high-risk class II HLA genotype have previously been identified as risk factors for type 1 diabetes $[2,14,22,23]$ and were therefore included in our analyses as fixed variables.

The suggested decision rules all provide very high specificities, although the sensitivities were relatively low. According to the current and previous [24] results, IFG in an OGTT is not suitable for predicting type 1 diabetes; although the opposite has also been implicated by Bleich et al [25], but based on a very small sample size of five individuals. By contrast, the detection of IGT provides a moderate sensitivity of $35 \%$ with a high specificity of $95 \%$ comparable to that of random plasma glucose $\geq 7.8 \mathrm{mmol} / \mathrm{l}$ with a sensitivity of $21 \%$ and a specificity of $94 \%$. Especially when taking into account the fact that in our study an OGTT was performed only once a year and random plasma glucose measured twice a year the results for prediction purposes are relatively good. Both IGT and random plasma glucose $\geq 7.8 \mathrm{mmol} / \mathrm{l}$ provide high HRs with a relatively short median time to diagnosis of type 1 diabetes (0.7 and 1.0 years, respectively). In future follow-up studies focusing on increasing the sensitivity of dysglycaemic markers, the frequency of random plasma glucose measurements should be increased and improved sensitivity might be obtained with a relatively modest effort. However, an increase in the number of measurements may affect the specificity; therefore, more studies are needed to demonstrate the utility of random plasma glucose in prediction and to identify the optimal number of samples per year to be measured in highrisk individuals. We anticipate that in the future the OGTT could potentially serve as a confirmatory test for dysglycaemia detected by simple and low-cost random plasma glucose measurements. 
Retrospective analysis in progressors and non-progressors showed an increase in 120 min glucose values in the OGTT and random plasma glucose concentrations 1.5 years before the presentation of clinical disease, reflecting the gradual deterioration in endogenous insulin secretion. Slowly progressing glucose intolerance was observed in previous studies as early as 1987 [26]. Progressors and nonprogressors showed differences in the age at seroconversion to autoantibody positivity and development of multiple islet autoantibodies. In addition, non-progressors less often had affected family members. These differences might interfere with the observed plasma glucose levels. However, our data show that the mean values of fasting and $2 \mathrm{~h}$ plasma glucose in OGTT as well as random plasma glucose concentrations remained very stable in the non-progressors during the whole follow-up period and therefore these values are considered to be suitable for comparison.

Our study is the first to describe the progression of dysglycaemia in a general population-derived cohort with a large number of children observed from birth to the diagnosis of type 1 diabetes. With a large sample size we were able to provide accurate and reliable estimates for HR, sensitivity, specificity and median time to diagnosis when analysing various markers of dysglycaemia in predicting type 1 diabetes. The study population came from three clinical centres with slightly different methods of glucose analysis, which might have affected the absolute values. However, the differences in laboratory methods were taken into account in the statistical analyses.

Sosenko and co-workers have studied the impact of the OGTT in two separate study populations, one derived from the Diabetes Prevention Trial-Type 1 (DPT-1) and the other from the TrialNet Natural History Study (TNNHS), both comprising autoantibody-positive relatives of patients with established type 1 diabetes [27]. The exact time of seroconversion or development of multiple autoantibodies and occurrence of dysglycaemia remain uncertain in that setting, and accordingly our population-derived study provides new and important information for disease prediction. According to their studies, a risk score derived from the C-peptide and glucose concentrations in the OGTT, BMI and age [28] provided a 2 year risk of diabetes of more than $75 \%$ in the population with the highest risk score $(\geq 9.00)$. However, only a relatively small proportion of the participants studied exceeded that value before diagnosis [7]. The 2 year risks of the DPT score [29] and IGT in our study did not practically differ from the risk associated with a single random plasma glucose $\geq 7.8 \mathrm{mmol} / 1$. According to our study, single random plasma glucose appears to be almost as good a predictor of type 1 diabetes as $2 \mathrm{~h}$ plasma glucose in an OGTT, although with somewhat lower sensitivity.

The time of the laboratory tests for random plasma glucose varied from 08:00 to 17:00 hours. The children and their families were not asked to prepare for the visit in any specific manner. Random plasma glucose is an easy and cost-efficient method to perform at the study site or even at home using a glucometer. For intervention studies aimed at preventing or postponing the development of clinical type 1 diabetes the identification of participants at high risk of developing type 1 diabetes is essential. OGTTs and the DPT risk score have been shown to identify high-risk children at relatively high cost. Random glucose monitoring could provide a practical way to identify optimal study populations for secondary prevention trials and also to minimise the risk of severe metabolic decompensation at the time of diagnosis. However, the optimal interval for sampling needs to be defined to improve the sensitivity of the test.

We have previously shown that the specificity of the first emerging autoantibody affects time from seroconversion to overt type 1 diabetes in a series of DIPP participants almost identical to the current one [30]. IA-2A as the primary autoantibody is associated with the shortest time to diagnosis, whereas progression after detection of IAA or GADA is similar. Seroconversion to IAA or IA-2A positivity occurs typically during the first years of life, while GADA appears later [30]. In the current study, IGT and a random plasma glucose $\geq 7.8 \mathrm{mmol} / 1$ occurred more frequently in children with IAA or IA-2A as the first autoantibodies when compared with those with GADA at seroconversion. This difference could be due to the design of the study, since the current study series involves mostly younger children leading to a reduced proportion of children having GADA as initial autoantibodies and presenting with type 1 diabetes during follow-up, which eventually affects the incidence of detected dysglycaemia. In the whole study group, $33 \%$ presented with two or more autoantibodies in their first antibody-positive sample and in that group the single primary autoantibody could not be identified. Further studies with longer follow-up especially in children with GADA as the primary autoantibody are needed to verify whether our observation of a higher frequency of dysglycaemia in children with IAA or IA$2 \mathrm{~A}$ as initial autoantibodies is true or is due to the study design.

According to our results, future follow-up studies should pay more attention to glucose monitoring in high-risk individuals. Random plasma glucose seems to be a practical, lowcost and specific biomarker for prediction of type 1 diabetes. These results also suggest that assessment of glucose metabolism provides important information that helps the clinician estimate the remaining time to clinical diagnosis and can be used when discussing with families with a child testing positive for multiple islet autoantibodies.

Acknowledgements We thank the dedicated personnel of the DIPP study in Oulu, Tampere and Turku; and the study children and their families for their essential contribution. 
Funding This work was supported by the following grants. International: JDRF International (grants 4-1998-274, 4-1999-731, 4-2001-435); European Union (grant BMH4-CT98-3314); Novo Nordisk Foundation. Finland: Academy of Finland (Centre of Excellence in Molecular Systems Immunology and Physiology Research 2012-2017, Decision No. 250114); TEKES National Technology Agency of Finland; Special Research Funds for University Hospitals in Finland; Finnish Office for Health Technology Assessment; Diabetes Research Foundation, Finland; Sigrid Juselius Foundation; Emil Aaltonen Foundation; Jalmari and Rauha Ahokas Foundation; Signe and Ane Gyllenberg Foundation; the Research Foundation of Orion Corporation; Foundation for Pediatric Research; Alma and KA Snellman Foundation; and Päivikki and Sakari Sohlberg Foundation.

Duality of interest The authors declare that there is no duality of interest associated with this manuscript.

Contribution statement $\mathrm{OH}$ had full access to all of the data in the study and takes responsibility for the integrity of the data and the accuracy of the data analysis. JI, OS, RV and MK contributed to the study concept and design. OH, SA, JI, OS, RV and MK contributed to the acquisition of the data. $\mathrm{OH}, \mathrm{SA}, \mathrm{TP}, \mathrm{RV}$ and MK contributed to the analysis and interpretation of the data and drafted the manuscript. OH, SA, TP, JI, OS, RV and MK critically revised the manuscript for important intellectual content. TP was responsible for the statistical analysis. OH, TP, JI, RV and MK provided administrative, technical or material support. RV and MK supervised the study. All authors read and approved the final version of the manuscript.

\section{References}

1. DIAMOND Project Group (2006) Incidence and trends of childhood type 1 diabetes worldwide 1990-1999. Diabet Med 23:857-866

2. Harjutsalo V, Sjoberg L, Tuomilehto J (2008) Time trends in the incidence of type 1 diabetes in Finnish children: a cohort study. Lancet 371:1777-1782

3. Siljander HT, Simell S, Hekkala A et al (2009) Predictive characteristics of diabetes-associated autoantibodies among children with HLA-conferred disease susceptibility in the general population. Diabetes 58:2835-2842

4. Ziegler AG, Rewers M, Simell O et al (2013) Seroconversion to multiple islet autoantibodies and risk of progression to diabetes in children. JAMA 309:2473-2479

5. Siljander HT, Hermann R, Hekkala A et al (2013) Insulin secretion and sensitivity in the prediction of type 1 diabetes in children with advanced beta-cell autoimmunity. Eur J Endocrinol 169:479-485

6. Sosenko JM, Skyler JS, Herold KC, Palmer JP, Type 1 Diabetes TrialNet and Diabetes Prevention Trial-Type 1 Study Groups (2012) The metabolic progression to type 1 diabetes as indicated by serial oral glucose tolerance testing in the Diabetes Prevention Trial-Type 1. Diabetes 61:1331-1337

7. Sosenko JM, Skyler JS, Mahon J et al (2012) The application of the Diabetes Prevention Trial-Type 1 Risk Score for identifying a preclinical state of type 1 diabetes. Diabetes Care 35:1552-1555

8. Ferrannini E, Mari A, Nofrate V, Sosenko JM, Skyler JS, DPT-1 Study Group (2010) Progression to diabetes in relatives of type 1 diabetic patients: mechanisms and mode of onset. Diabetes 59:679-685

9. Sosenko JM, Palmer JP, Greenbaum CJ et al (2006) Patterns of metabolic progression to type 1 diabetes in the Diabetes Prevention Trial-Type 1. Diabetes Care 29:643-649
10. Helminen O, Aspholm S, Pokka T et al (2015) $\mathrm{HbA}_{1 \mathrm{c}}$ predicts time to diagnosis of type 1 diabetes in children at risk. Diabetes 64:1719-1727

11. Elding Larsson H, Vehik K, Gesualdo P et al (2014) Children followed in the TEDDY study are diagnosed with type 1 diabetes at an early stage of disease. Pediatr Diabetes 15:118-126

12. Triolo TM, Chase HP, Barker JM, DPT-1 Study Group (2009) Diabetic subjects diagnosed through the Diabetes Prevention Trial-Type 1 (DPT-1) are often asymptomatic with normal $\mathrm{A}_{1 \mathrm{c}}$ at diabetes onset. Diabetes Care 32:769-773

13. Barker JM, Goehrig SH, Barriga K et al (2004) Clinical characteristics of children diagnosed with type 1 diabetes through intensive screening and follow-up. Diabetes Care 27:1399-1404

14. Alberti KG, Zimmet PZ (1998) Definition, diagnosis and classification of diabetes mellitus and its complications. Part 1: diagnosis and classification of diabetes mellitus. Provisional report of a WHO consultation. Diabet Med 15:539-553

15. Sosenko JM, Palmer JP, Rafkin-Mervis L et al (2009) Incident dysglycemia and progression to type 1 diabetes among participants in the Diabetes Prevention Trial-Type 1. Diabetes Care 32: 1603-1607

16. Beer SF, Heaton DA, Alberti KG, Pyke DA, Leslie RD (1990) Impaired glucose tolerance precedes but does not predict insulindependent diabetes mellitus: a study of identical twins. Diabetologia 33:497-502

17. Hermann R, Knip M, Veijola R et al (2003) Temporal changes in the frequencies of HLA genotypes in patients with type 1 diabetes-indication of an increased environmental pressure? Diabetologia 46:420-425

18. Hekkala A, Ilonen J, Knip M, Veijola R, Finnish Paediatric Diabetes Register (2011) Family history of diabetes and distribution of class II HLA genotypes in children with newly diagnosed type 1 diabetes: effect on diabetic ketoacidosis. Eur J Endocrinol 165:813-817

19. Report WHO (2006) Definition and diagnosis of diabetes mellitus and intermediate hyperglycemia. WHO Document Production Services, Geneva

20. Siljander HT, Veijola R, Reunanen A, Virtanen SM, Akerblom HK, Knip M (2007) Prediction of type 1 diabetes among siblings of affected children and in the general population. Diabetologia 50: 2272-2275

21. Aly TA, Ide A, Jahromi MM et al (2006) Extreme genetic risk for type 1A diabetes. Proc Natl Acad Sci U S A 103:14074-14079

22. Parikka V, Nanto-Salonen K, Saarinen M et al (2012) Early seroconversion and rapidly increasing autoantibody concentrations predict prepubertal manifestation of type 1 diabetes in children at genetic risk. Diabetologia 55:1926-1936

23. Nanto-Salonen K, Kupila A, Simell S et al (2008) Nasal insulin to prevent type 1 diabetes in children with HLA genotypes and autoantibodies conferring increased risk of disease: a double-blind, randomised controlled trial. Lancet 372:1746-1755

24. Greenbaum CJ, Cuthbertson D, Krischer JP, Disease Prevention Trial of Type I Diabetes Study Group (2001) Type I diabetes manifested solely by 2 -h oral glucose tolerance test criteria. Diabetes 50:470-476

25. Bleich D, Jackson RA, Soeldner JS, Eisenbarth GS (1990) Analysis of metabolic progression to type I diabetes in ICA+ relatives of patients with type I diabetes. Diabetes Care 13:111-118

26. Tarn AC, Smith CP, Spencer KM, Bottazzo GF, Gale EA (1987) Type I (insulin dependent) diabetes: a disease of slow clinical onset? Br Med J (Clin Res Ed) 294:342-345

27. Sosenko JM, Mahon J, Rafkin L et al (2011) A comparison of the baseline metabolic profiles between Diabetes Prevention TrialType 1 and TrialNet Natural History Study participants. Pediatr Diabetes 12:85-90 
28. Sosenko JM, Krischer JP, Palmer JP et al (2008) A risk score for type 1 diabetes derived from autoantibody-positive participants in the Diabetes Prevention Trial-Type 1. Diabetes Care 31:528-533

29. Sosenko JM, Skyler JS, Mahon J et al (2014) Use of the Diabetes Prevention Trial-Type 1 Risk Score (DPTRS) for improving the accuracy of the risk classification of type 1 diabetes. Diabetes Care 37:979-984

30. Ilonen J, Hammais A, Laine AP et al (2013) Patterns of beta-cell autoantibody appearance and genetic associations during the first years of life. Diabetes 62:3636-3640 